
\title{
Yield of South Korean Pig Carcasses Using Machine Vision Technique
}

Santosh Lohumi ${ }^{1}$, Collins Wakholi ${ }^{1}$, Jong Ho Baek ${ }^{2}$, Byeoung Do Kim², Se Joo Kang ${ }^{2}$, Hak Sung Kim², Yeong Kwon Yun², Wang Yeol Lee², Sung Ho Yoon², and Byoung-Kwan $\mathrm{Cho}^{1, *}$

${ }^{1}$ Department of Biosystems Machinery Engineering, College of Agricultural and Life Science, Chungnam National University, Daejeon 34134, Korea

${ }^{2}$ Korea Institute for Animal Products Quality Evaluation, Sejong 30100, Korea

*Corresponding author : Byoung-Kwan Cho Department of Biosystems Machinery Engineering, College of Agricultural and Life Science, Chungnam National University,

Daejeon 34134, Korea

Tel: $+82-42-821-6715$

Fax: +82-42-823-6246

E-mail: chobk@cnu.ac.kr

\section{*ORCID}

Santosh Lohumi

https://orcid.org/0000-0002-5437-2411

Collins Wakholi

https://orcid.org/0000-0003-0635-9463

Byeoung Do Kim

https://orcid.org/0000-0001-8622-0482

Se Joo Kang

https://orcid.org/0000-0003-1192-3399

Hak Sung Kim

https://orcid.org/0000-0002-9162-5967

Yeong Kwon Yun

https://orcid.org/0000-0002-8374-3525

Wang Yeol Lee

https://orcid.org/0000-0003-1281-3781

Sung Ho Yoon

https://orcid.org/0000-0003-0236-4492

Byoung-Kwan Cho

https://orcid.org/0000-0002-8397-9853

\section{Erratum}

In the published article "Nondestructive Estimation of Lean Meat Yield of South Korean Pig Carcasses Using Machine Vision Technique. Korean J Food Sci Anim Resour 38:1109-1119. https://doi.org/10.5851/kosfa.2018.e44," some sentences have to be modified. At the request of the author, the editorial office have confirmed that there were some mistakes in the use of abbreviations and units. We attach the pdf that reflects the modification.

\section{Supplementary Materials}

Modified article is only available online from: https://oi.org/10.5851/kosfa.2019.e47

\section{References}

Lohumi S, Wakholi C, Baek JH, Kim BD, Kang SJ, Kim HS, Yun YK, Lee WY, Yoon SH, Cho BK. 2018. Nondestructive estimation of lean meat yield of south Korean pig carcasses using machine vision technique. Korean J Food Sci Anim Resour 38:1109-1119.

(C) Korean Society for Food Science of Animal Resources. This is an open access article distributed under the terms of the Creative Commons Attribution Non-Commercial License (http://creativecommons.org/licences/by-nc/3.0) which permits unrestricted non-commercial use, distribution, and reproduction in any medium, provided the original work is properly cited. 
OPEN ACCESS

$\begin{array}{ll}\text { Received } & \text { September 10, } 2018 \\ \text { Revised } & \text { October 3, 2018 } \\ \text { Accepted } & \text { October 4, } 2018 \\ \text { Modifed } & \text { June 30, } 2019\end{array}$

\footnotetext{
*Corresponding author : Byoung-Kwan Cho Department of Biosystems Machinery

Engineering, College of Agricultural and Life

Science, Chungnam National University,

Daejeon 34134, Korea

Tel: $+82-42-821-6715$

Fax: $+82-42-823-6246$

E-mail: chobk@cnu.ac.kr
}

\author{
Santosh Lohumi ${ }^{1}$, Collins Wakholi ${ }^{1}$, Jong Ho Baek ${ }^{2}$, Byeoung Do Kim², Se Joo \\ Kang ${ }^{2}$, Hak Sung Kim², Yeong Kwon Yun², Wang Yeol Lee², Sung Ho Yoon², \\ and Byoung-Kwan $\mathrm{Cho}^{1, *}$
}

${ }^{1}$ Department of Biosystems Machinery Engineering, College of Agricultural and Life Science, Chungnam National University, Daejeon 34134, Korea

${ }^{2}$ Korea Institute for Animal Products Quality Evaluation, Sejong 30100, Korea

\begin{abstract}
In this paper, we report the development of a nondestructive prediction model for lean meat yield in Korean pig carcasses and in the major cuts using a machine vision technique. A popular vision system in the meat industry, the VCS2000 was installed in a modern Korean slaughterhouse, and the images of half carcasses were captured using three cameras from 175 selected pork carcasses ( 86 castrated males and 89 females). The imaged carcasses were divided into calibration $(\mathrm{n}=135)$ and validation $(\mathrm{n}=39)$ sets and a multilinear regression (MLR) analysis was utilized to develop the prediction equation from the calibration set. The efficiency of the prediction equation was then evaluated by an independent validation set. We found that the prediction equation-developed to estimate lean meat percentage (LMP) in whole carcasses based on six variables-was characterized by a coefficient of determination $\left(R_{v}^{2}\right)$ value of 0.77 (root-mean square error [RMSEV] of $2.12 \%$ ). In addition, the predicted lean meat yield in the major cuts: ham, belly, and shoulder exhibited $R_{v}^{2}$ values $\geq 0.8$ ( 0.73 for loin parts) with low RMSEV values. However, lower accuracy $\left(R_{v}^{2}=0.67\right)$ was achieved for tenderloin cuts. These results indicate that the LMP in Korean pig carcasses and major cuts can be predicted successfully using the VCS2000-based prediction equation developed here. The ultimate advantages of this technique are compatibility and speed, as the VCS2000 imaging system can be installed in any slaughterhouse with minor modifications to facilitate the on-line and real-time prediction of lean meat yield in pig carcasses.
\end{abstract}

Keywords pork grading, lean meat percentage, quality measurement, VCS2000, automation

\section{Introduction}

The level of pork consumption in Korea ranked seventh in the world and third in Asia (Choe et al., 2015). Due to increasing consumption of pork in Korea, it has become necessary for the governing bodies and regulatory organizations to obtain objective data

(C) Korean Society for Food Science of Animal Resources. This is an open access article distributed under the terms of the Creative Commons Attribution Non-Commercial License (http://creativecommons.org/licences/by-nc/3.0) which permits unrestricted non-commercial use, distribution, and reproduction in any medium, provided the original work is properly cited. 
for pork production to maintain a stable national supply of pork. The production can be estimated by measuring the lean meat percentage (LMP) of slaughtered carcasses in slaughter houses across the country. Thus, based on the estimated LMP, breeders can be encouraged to breed more productive (muscular) animals according to the need and trends of the consumer's market (Savescu and Laba, 2016). Moreover, pork import (to Korea) can be forecasted based on the estimated LMP value and market demand.

A range of techniques is available to estimate the lean meat percentage of pig carcasses. The United States Department of Agriculture (USDA) standard equation — based on hot carcass weight, $10^{\text {th }}$ rib backfat depth, and the $10^{\text {th }}$ rib loin area-has been previously used to predict the lean and fat composition of pork carcass (Forrest et al., 1989). Owing to the modernization of slaughterhouses, which aim to process a larger number of carcasses, and because of differences in legislation from countries around the globe, a wide range of techniques has been evaluated in the last decade for the rapid and automated on-line measurement of pork carcass composition. These techniques include the Fat-O-Meat'er (FOM), the UltraFOM (UFOM), the AutoFOM, and a vision-based video image analyzer (VCS2000) (Font I Furnols and Gispert, 2009). FOM is a handheld optical probe that measure backfat thickness and loin muscle depth in predefine location on the carcass to predict the lean meat content (Lucas et al., 2017). However, equipment such as UltraFOM and AutoFOM use reflectance of ultrasound to measure the lean meat percentage and fat thickness. UltraFOM is handled manually and AutoFOM is automatic (Olsen et al., 2007). Whereas, VCS2000 is a vision based technique for image processing based automatic detection in lean meat percentage in half carcass (Daumas and Causeur, 2008).

Most of country has a system in place to control the price paid to producers for their pork, where a measuring device (either manual or automated) takes measurements and calculates the LMP by means of a prediction equation. However, Korea still does not use automated techniques for pig classification and the real-time and rapid estimation of LMP in pig carcass. Very few modern slaughterhouses use semi-automated techniques (UltraFom 300), while medium and low-scale slaughterhouses still depend on time-consuming conventional methods such as standard dissection methods and by following USDA standard equation as mentioned above (Forrest et al., 1989).

At present, there are a range of instruments available for the estimation of LMP, and thus for the determination of the market value of pork carcasses. Likewise, a variety of studies have been performed to compare the performance of semiautomated (Fat-O-meter and UFOM) and fully-automated (AutoFOM and VCS2000) devices in measuring LMP (Branscheid and Dobrowolsk, 2000; Font I Furnols et al., 2004; Font I Furnols and Gispert, 2009). The results of these studies are comparable based on the root mean square errors of prediction (RMSEP), where some instruments have been shown to yield a low RMSEP for the estimation of LMP of the whole carcass, and some appear to be effective in estimating the LMP of primal cuts. However, the VCS2000 has the advantage of being automated, and is currently being used (along with other aforementioned techniques) in most slaughterhouses across Europe (Daumas and Causeur, 2008; Engel et al., 2006; Gispert and Font I Furnols, 2012).

In order for the VCS2000 system to be effective in estimating the LMP of pork carcasses, it requires a calibration equation (prediction formula), which is developed from the parameters of the collected images (i.e., variables measured by VCS2000). However, it is not practical to use existing calibration equations (from European countries) in Korea, as the calibration equation is highly dependent on pork variety (i.e., Korean pigs are genetically different from European pigs), and variations in a given population (i.e., sex) (Font I Furnols et al., 2004). Moreover, pigs from Korea and European Union (EU) have different wholesale and retail cuts.

Therefore, the main objective of the present work was to introduce a pork carcass grading system (based on LMP) to 
Korean slaughterhouses in order to improve the work efficiency and the speed of pork processing operations. To achieve this objective, we sought to: (1) install a VCS2000-based system for the on-line estimation of the LMP of pork carcasses in a rapid manner, and (2) develop a calibration equation for the estimation of LMP in Korean whole pork carcasses and lean meat yield of their primal cuts. The calibration equation developed here can be further used by other Korean slaughterhouses in the future.

\section{Materials and Methods}

This investigation was conducted on 175 carcasses of diverse origins, genetic groups, and production environments, and is thus considered to be representative of the national pig population of Korea. In order to determine the prediction equation for the estimation of the lean meat percentage of whole pig carcasses and major cuts using the VCS 2000 imaging system, an experiment was carried out from April to September 2016 in a recently developed modern slaughterhouse located in the Gyeongbuk province of Korea.

\section{Carcass sampling}

A total of 175 carcasses - representing variation in breeds on a national level—were dissected and used in this experiment. Out of 175 total carcasses, 86 were castrated males and 89 were females. The selected carcasses were composed of a wide range of weights, meat yield, and a range of grades in order to accurately estimate the parameters of the prediction equation. The objective here was to analyze a sufficient number of carcasses at the extremes, thereby ensuring an accurate estimation of lean meat percentage for all weights, genders, and grades (level of fattiness). The descriptive statistics of carcasses used in the present study are presented in Table 1. All carcasses selected for the present study were bled and eviscerated, and divided down the midline into two equal halves following the Korean reference dissection methods. The differences in weight between the left and right halves of the carcasses were within $1 \%$ of the whole carcass weight, where carcasses with $>1 \%$ differences in the weights of the two halves were not selected for imaging. All half carcasses were refrigerated for $24 \mathrm{~h}$, where the average weight loss after refrigeration was approximately $2.5 \%$ of the initial weight of half carcasses.

\section{VCS2000 image acquisition and analysis system}

The VCS2000 system was installed in a slaughterhouse for the on-line and automated prediction of LMP in half carcasses and lean meat yield in primal cuts. As VCS2000 is a vision system that is able to measure fat depth, muscle thickness, area, and the ratios between these parameters, it generates a large number of variables.

Table 1. Descriptive statistics of carcasses

\begin{tabular}{lcccccccc}
\hline & & \multicolumn{3}{c}{ Hot carcass weight $(\mathrm{kg})$} & & & \multicolumn{3}{c}{ LMP $\%$} \\
\cline { 3 - 5 } Measure $(\mathrm{n}=175)$ & Total number & Mean & Min-Max & SD & & Mean & Min-Max & SD \\
Male carcasses & 86 & 86.12 & $62-104.2$ & 10.49 & & 54.5 & $42.36-65.38$ & 4.58 \\
Female carcasses & 89 & 85.93 & $68.7-104.9$ & 10.27 & & 56.32 & $43.89-67.55$ & 4.94 \\
Calibration set & 135 & 86.18 & $62-104.2$ & 10.12 & & 55.38 & $42.36-67.55$ & 4.96 \\
Validation set & 39 & 85.48 & $68.7-104.9$ & 10.91 & & 55.45 & $46.13-62.14$ & 4.48 \\
\hline
\end{tabular}

One of the carcasses of this group was considered outlier and removed from all the calculation. LMP, lean meat percentage. 
One monochromatic and two color cameras were used as the vision sensors for image acquisition. Two light sources were placed alongside the camera at a distance of approximately $1,800 \mathrm{~cm}$ to insure the proper illumination of the carcasses. In addition, the field of view (FOV; $5 \mathrm{~m}$ long $\times 3 \mathrm{~m}$ wide) of the camera was secured along the length of the slaughter line to prevent any image overlapping. According to the manufacturer instructions for the VCS2000, the carcass half was positioned in the front of each camera to record data. As carcasses moved along the slaughter line, the hanged half carcass first came into the FOV of the monochromatic camera, which collected images and took measurements of the upper side of the carcass based on the position of the hind leg on the exterior part of the carcass. Subsequently, the carcass moved forward and the two color cameras at the second camera station to collected images of the upper side, the lower side, and the interiors of the half carcass. The image data were then sent to a computer installed in the slaughterhouse, and the data were processed using special image-processing software. A schematic that summarizes image processing and feature extraction for the VCS2000 system is shown in Fig. 1.

The proprietary image processing software consists of 3 major sections, which includes image processing, feature detection and extraction, and feature selection (summarized in Fig. 2). During image processing, the operations performed on the acquired images include image stitching, background removal, estimation of outlines, ROI detection, etc. After image processing, image feature detection and extraction include algorithms for: the robust detection of key points, the determination of features from key-points and lines, and the combination of features to form new variables. Feature extraction is followed by feature selection, which makes use of the actual LMP values together with all the extracted features to find several cardinal features that are later used in the prediction of LMP.

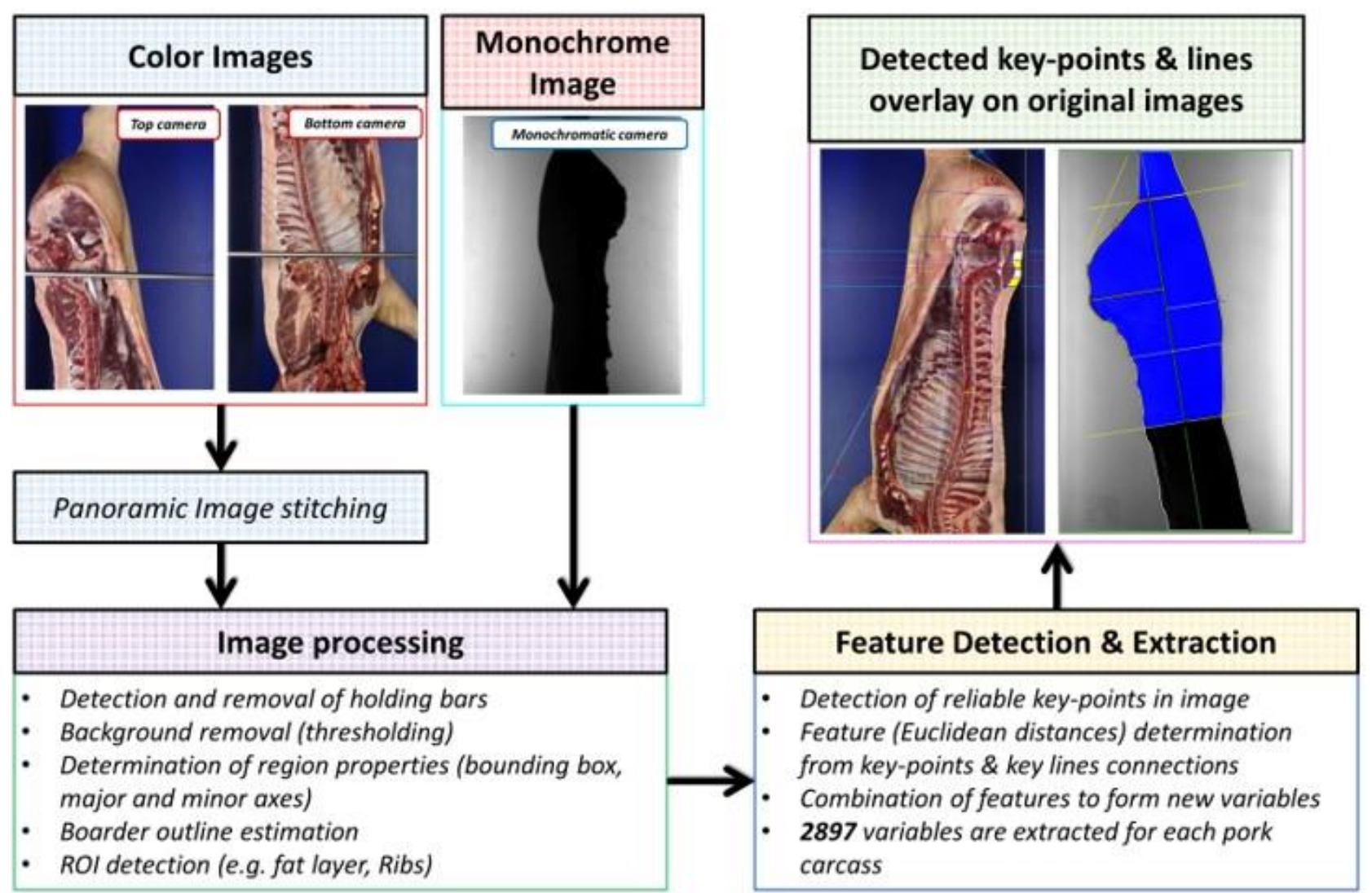

Fig. 1. Summary of image processing and feature selection for the VCS2000 system. The images shown were partially adopted from www.eplusv.de. 


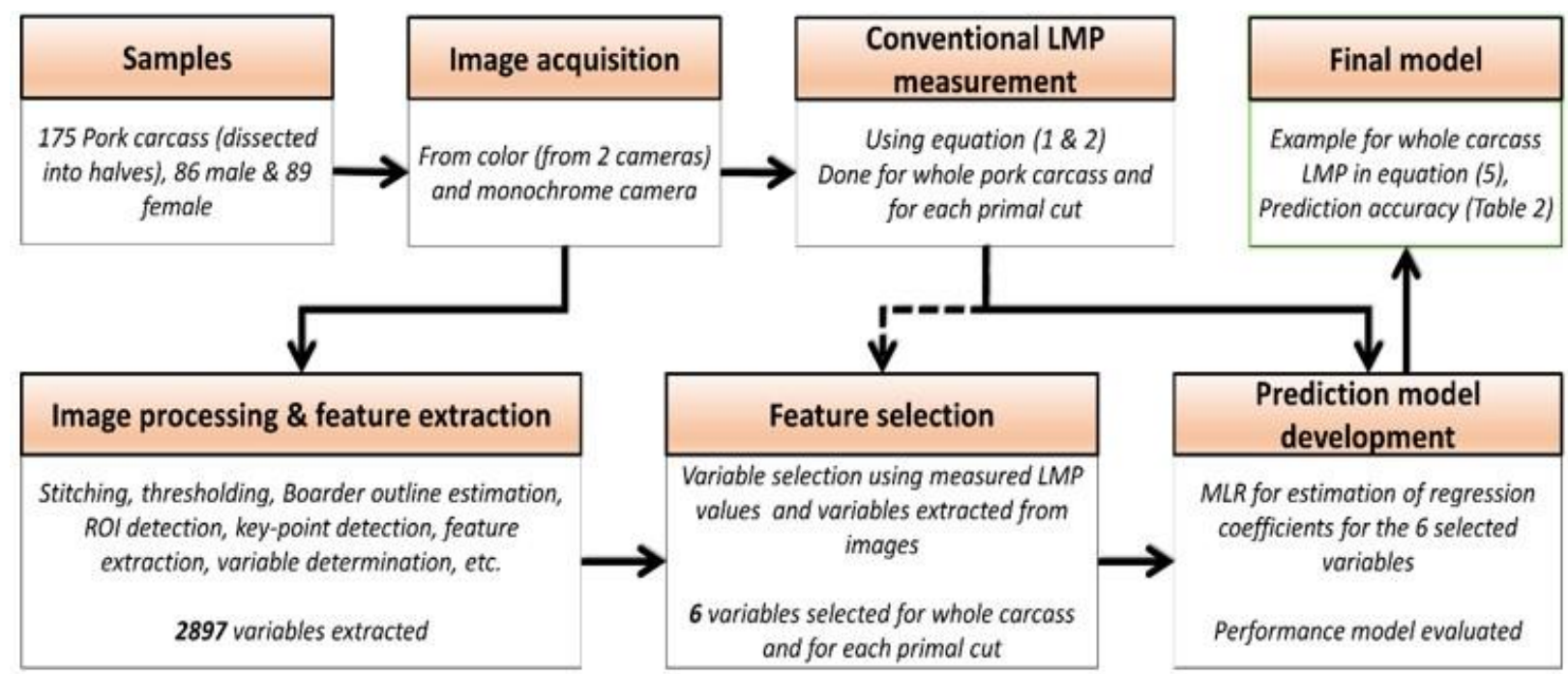

Fig. 2. Schematic for estimation of pork lean meat percentage (LMP) using the VCS2000.

The weight and backfat thickness of each carcass half were measured and a unique identity number was assigned. In general, in the EU, the identity number is usually painted in red color in the sirloin region. However, in the present study, in order to reduce any color-based interference in the images, and considering the high consumer demand for sirloin, we instead marked the hip. These external parameters (weight, backfat thickness, and identity number) were input into the system. In order to improve accuracy and remove bias, four independent and experienced classifiers, invited from four different institutes, carried out the measurements of backfat thickness.

\section{Carcass cutout and calculation of lean meat percentage}

After imaging, half carcasses were cut into pieces and dissected according to a pre-defined cut test protocol by four dissection experts. As the Korean reference dissection method differs slightly from the simplified European reference dissection method, and as the installed system (VCS2000) was initially optimized for use in EU countries, two extra carcasses were first dissected by a team of four trained professionals. Finally, upon the acceptance of Korean dissection methods by the system manufacturer, we proceeded with dissections to fulfill the objectives of the experiments. Each carcass was first dissected into major cuts by all four professionals and then lean meat content from each part of the carcass was extracted by one person for each carcass. We determined that there were no significant differences in the total lean meat content or in major cuts between dissection personnel. The reference lean meat content of the carcass was calculated using the lean meat from of the major cuts according to the definitions of the European regulation commission (European Commission, 2014) as follows:

LMP089 (\%) $=0.89 \frac{\Sigma \text { Lean }(\text { ham, loin, belly, shoulder })+\text { tenderloin }}{\sum \text { Weight }(\text { ham }, \text { loin, belly, shoulder, tenderloin })} \times 100$

The LMP of the different cuts was obtained as follows:

LMP cut $(\%)=\frac{\text { Lean of the cut }}{\text { Weight of the cut }} \times 100$ 


\section{Statistical analyses}

From the images collected from half carcass (using three cameras), the VCS2000 produced a total of 2897 variables (features). However, considering the computation speed necessary for the real time prediction of lean meat content in half carcass and major cuts, it is not feasible to use all 2897 of the variables. Therefore, a total of 6 variables were first selected to be optimal variables using the VCS image processing software provided by the system manufacturer. Subsequently, the prediction formula for the LMP was derived by multilinear regression (MLR) using the selected variables. In order to develop a legitimate MLR-based equation, imaged carcass samples were divided into calibration and validation sets considering the four main factors: gender, weight, lean meat content, and measured back-fat thickness. In the MLR equation, the measured LMP served as dependent variables and the six VCS-selected variables served as independent variables. The MLR analysis was carried out using SAS software (SAS Institute, Cary, NC, USA).

The RMSE and coefficient of determination $\left(\mathrm{R}^{2}\right)$ for both calibration and validation sets were adopted as goodness of fit criteria. In the past (according to EU regulations), accuracy was expressed in the form of the relative standard deviation (RSD) of the linear regression, and on by the RMSE in order to incorporate prediction error due to possible bias (Engle et al., 2006). The RMSE was derived by the following expression, were summation in over the carcasses (n) involved in the calculations:

$$
R M S E=\sqrt{\sum \frac{r^{2}}{n}}
$$

where $r$ is the residual calculated by subtracting the MLR based predicted value from the reference values of LMP.

The $\mathrm{R}^{2}$ values for both calibration and validation sets were calculated based on the measured $(y)$ and predicted lean meat content $(\hat{y})$ as follow:

$$
R^{2}=\frac{\sum(\hat{y}-\bar{y})^{2}}{\sum(y-\bar{y})^{2}}
$$

An overview of the operations and processes of the VCS2000 for the estimation of LMP in Korean pork during the experiment are summarized in Fig. 2 below. Details on how the VCS2000 system operates (especially the algorithms) were protected by the manufacturer.

\section{Results and Discussion}

According to the legislation of the EU, a dissection experiment must be conducted on a representative sample of at least 120 pig carcasses (Savescu and Laba, 2016), and it is necessary to have an error of prediction (RMSEP) lower than $2.5 \%$ (Font I Furnols and Gispert, 2009) in order to obtain authorization for the calibration equation to be used to calibrate each device, hence the calibration equation was developed with 135 carcasses. The descriptive statistics for the calibration and validation sets are given in Table 1, which illustrates the similar distributions of the four aforementioned factors in both the calibration and validation data sets. Although carcass weight was not significantly different ( $>0.05$ ) between male (average $86.12 \mathrm{~kg}$ and) and female (average $85.93 \mathrm{~kg}$ ) carcasses, the LMP of female carcasses (average 56.32\%) was slightly higher 
than that of male carcasses (average 54.5\%) (Table 1). In addition, the lean meat weight of the major cuts after dissection were comparable between the male and female carcasses.

\section{Prediction formula for carcass LMP}

The multivariate analytical model of the multiple linear regression (MLR) analysis was conducted on the 175 sample carcasses, using the six variables selected by the VCS2000 as predictors (independent variables) and the dissected LMP of the carcasses as dependent variables (reference values). However, before the development of the calibration equation, it was crucial to first check for outliers in the data set to ensure the robustness of the model and calibration equation. Outliers are defined as observations that are numerically distant from the rest of the data (Rahman et al., 2012). Therefore, to detect outlying observations, we inspected the residual plot and the range of the prediction variables. Only one carcass was selected as outlier, and was therefore not included in the development of the calibration equation for the prediction of LMP.

The results from the MLR analysis for both calibration and validation sets are summarized in Table 2. The results of the MLR obtained from the quantitative analysis for the LMP prediction of whole carcass data for both the calibration and validation sets, which was developed using the six variables, exhibit a strong relationship between the actual and predicted LMP concentration (coefficient of determination $\left[\mathrm{R}^{2}\right]$ values of 0.82 and 0.77 and RMSE values of $2.09 \%$ and $2.12 \%$ for the calibration and validation sets, respectively). Fig. 3A shows the prediction plot of lean meat content, where most points fall on the regression line. However, a few samples from the calibration set with low values for LMP exhibit slightly larger deviations from the regression line. Fig. 3B demonstrates that the residuals were randomly dispersed throughout the $x$-axis (actual LMP), and therefore have constant variance. The residuals for the validation set were tightly grouped and typically had a calculated RSD value of $2.41 \%$.

The calibration equation (prediction formula) for the prediction of LMP in whole carcasses, as obtained by multiple linear regression, is as follows:

Total LMP (whole carcass)

$$
\begin{aligned}
& =-0.0007578 \times H L 8^{2}-285.6022444 \times \frac{H L 15}{T L 5}-34.9153123 \times \frac{H L 29}{T L 1}+0.3184768 \\
& \times S B A R 1-0.2864066 \times K B A M 5+0.3820551 \times H L 29+86.4018008 \ldots \ldots \ldots \ldots \ldots \ldots \ldots \ldots \ldots \ldots \ldots
\end{aligned}
$$

The prediction formula presented above was derived ignoring the sex of the pig and is applicable to a wide variety of Korean pork, as the RMSEP was calculated based on all carcasses in the validation set $(n=39)$. In addition, the error value of

Table 2. Accuracy of lean meat prediction in whole carcass and major cuts using the VCS2000 and multiple linear regression analysis

\begin{tabular}{lllcccc}
\hline Parameters & Ham & Loin & Belly & Shoulder & Tenderloin & Whole carcass \\
$R_{c}^{2}$ & 0.87 & 0.85 & 0.91 & 0.90 & 0.77 & 0.82 \\
RMSEC & 0.33 & 0.19 & 0.26 & 0.16 & 0.03 & 2.09 \\
$R_{v}^{2}$ & 0.80 & 0.73 & 0.89 & 0.85 & 0.67 & 0.77 \\
RMSEV & 0.39 & 0.25 & 0.29 & 0.21 & 0.04 & 2.12 \\
\hline
\end{tabular}

RMSEC and RMSEP values for major cuts are in $\mathrm{kg}$ and in \% for whole carcass.

RMSEC, root mean square errors of calibration; RMSEP, root mean square errors of prediction. 
(A)

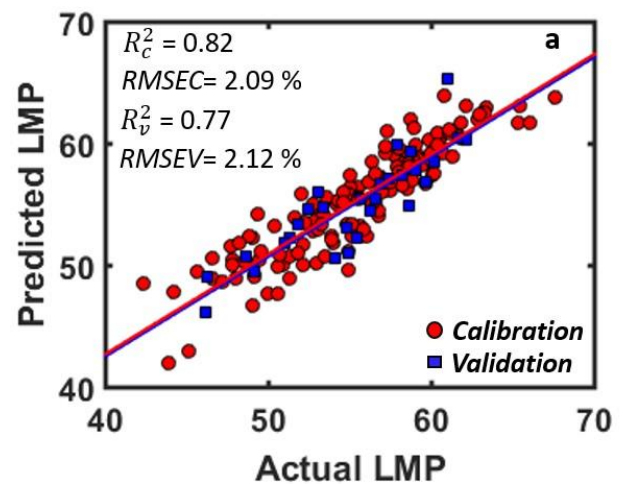

(B)

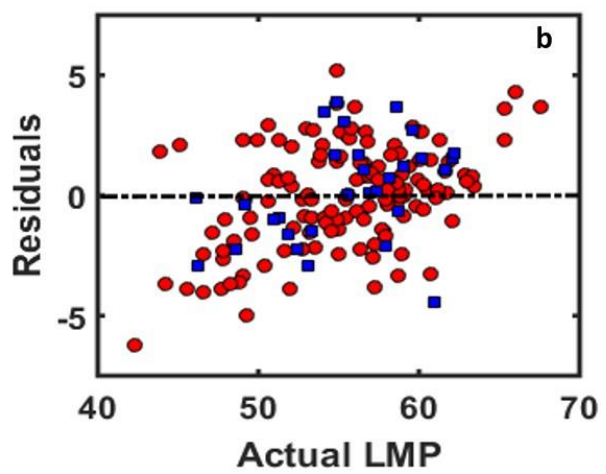

Fig. 3. Actual versus predicted percentage of lean meat for whole carcass (A) and the corresponding residual plot (B).

this calculation $(2.12 \%)$ is much lower than the $2.5 \%$ cut-off, and therefore meets the standard requirement.

\section{Prediction of lean meat yield in the major cuts}

Pork is usually sold in seven major cuts, where the different cuts are preferred by consumers from various countries. For example, pork belly is the most popular cut in Korea in comparison to the other cuts (Kim and Kim, 2018). However, the estimation of total LMP does not give a complete picture about the value of each of the cuts. Therefore, in order to determine the market value of each of the major cuts, a separate prediction equation was developed for each cut and was validated in the present study. Based on our results, the VCS2000 allows for the highly accurate determination of the lean meat yield of the major cuts (ham, loin, belly, shoulder, and tenderloin). The accuracy $\left(R_{v}^{2}\right)$ and error of the prediction (RMSEV) between the commercial values of the major cuts analyzed via VCS2000 imaging and the manually dissected major cuts are presented in Table 2. As a result, we observed high $R_{v}^{2}$ and low RMSEV values for ham, belly, and shoulder cuts $\left(R_{v}^{2} \geq 0.8\right)$. The prediction equation for the prediction of lean meat of loin cuts exhibits a reasonable degree of accuracy, where $R_{v}^{2}=0.73$ and $\mathrm{RMSEP}=0.25$. However, no strong relationship was observed between the values for measured and predicted lean meat in tenderloin cuts, which exhibits a relatively low $R_{v}^{2}$ value (0.67). The higher error of prediction for the tenderloin could possibly be a result of an error in cutting and dissecting. Moreover, the lean meat content of the tenderloin cut was between 0.38 and $0.69 \mathrm{~kg}$, where even a small difference can introduce a high degree of error into the prediction equation. However, it should be noted that high accuracy and low error values were achieved for belly and shoulder cuts despite the fact that shoulder is the most difficult joint to cut and the belly is the most difficult area to dissect (Nissen et al., 2006). In addition, a similar analysis performed by Bae et al. (2016) that predicted the lean meat in Korean ham using an AutoFOM-based technique found a $\mathrm{R}^{2}$ of 0.72 . The $\mathrm{R}^{2}$ value for the validation set in the present study was much higher (0.8) using the VCS2000 imaging technique.

Fig. 4 outlines the performance of the developed prediction equation for VCS2000 imaging data for the half carcasses in the prediction of lean meat content in the major cuts. From Fig. 4 (top), it is apparent that most data points fall near the regression line; however, in the case of the tenderloin cut, the data points are more scattered. Fig. 4 (bottom) also shows plots for the detection of residuals against the actual values for lean meat in major cuts. The residual plots show lower residual error difference for the validation sets for ham, belly, and shoulder cuts, and exhibit calculated RSD values of $0.34 \mathrm{~kg}, 0.32$ $\mathrm{kg}$, and $0.23 \mathrm{~kg}$, respectively. However, the residual for the loin and tenderloin cuts are relatively higher and have RSD values of $0.28 \mathrm{~kg}$ and $0.047 \mathrm{~kg}$, respectively. A comparison of residual difference can be made easily by considering the values on Y- 


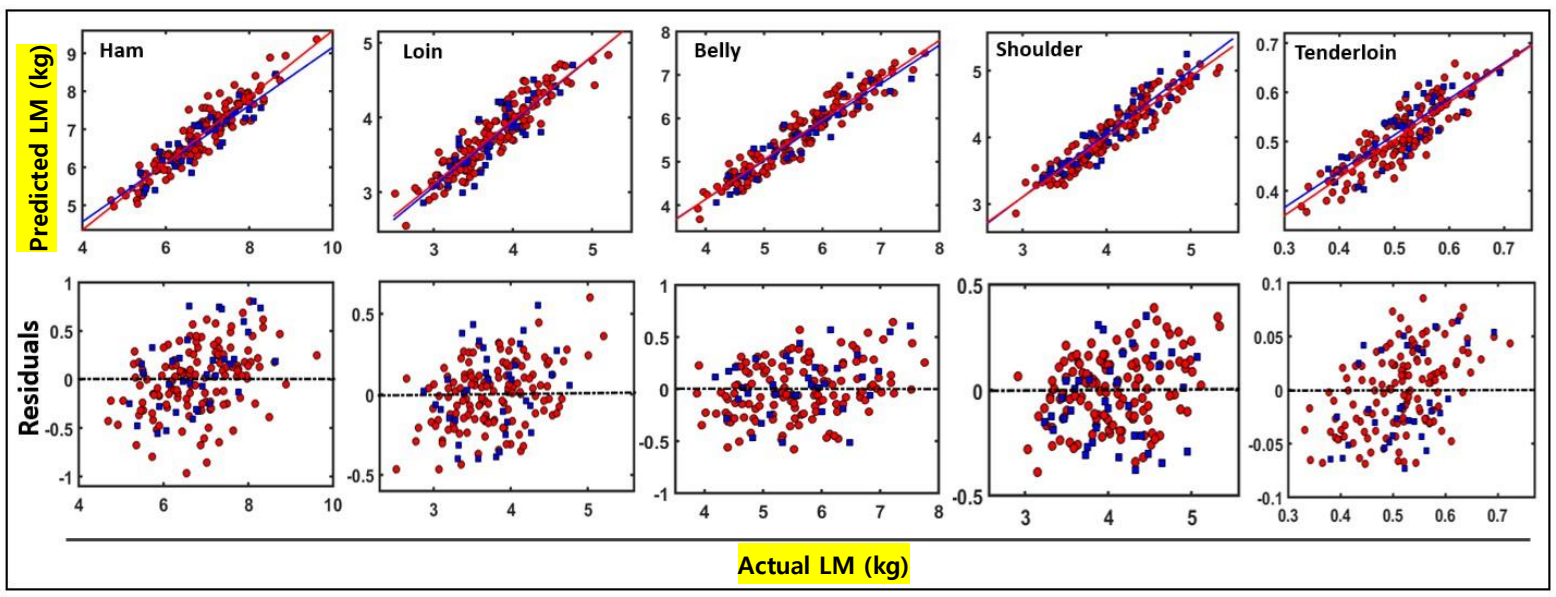

Fig. 4. Actual versus predicted values of lean meat in major cuts of carcasses (above) and the corresponding residual plots (below).

axis of prediction plot and on corresponding residual plot.

Apart from the prediction of LMP in whole carcasses and lean meat yield in the aforementioned major cuts, prediction equations were also developed for an imaging-based prediction of backfat thickness and predictions of lean meat in the neck, hind leg, and ribs. The prediction equation that was developed yielded $R_{v}^{2}$ and RMSEV values of 0.94 and $1.99 \mathrm{~mm}$ for backfat thickness, 0.84 and $0.12 \mathrm{~kg}$ for trimmed neck, 0.89 and $0.35 \mathrm{~kg}$ for hind leg, and 0.78 and $0.09 \mathrm{~kg}$ for ribs, respectively.

The prediction equation developed for Korean carcasses in the present study exhibit a higher degree of accuracy in comparison to the VCS2000-based prediction equation for the same purpose in the EU (Engle et al., 2006; Gispert and Font I Furnol, 2012). This is probably a result of differences in the two methods of dissection, where the Korean dissection method may be more accurate. Moreover, the VCS2000 is influenced by the accuracy of the splitting of the carcass, as it measures the interior part of the half carcass. In the present study, only well split carcasses were used for subsequent analyses. In addition, as previously mentioned, carcasses with more than a $1 \%$ difference in the weight of the two halves, or weight differences in the lean meat of the major cuts greater than $2 \%$ in both carcass sights were not included in the development of the prediction equation. Therefore, the resulting MLR model is a more robust and accurate prediction equation for the prediction of LMP in whole carcasses, as well as in major cuts. Furthermore, the wide range of carcasses (male and female) chosen for this experiment, as well as the relatively small degree of variation within each group, are a testament to the high level of care taken throughout this investigation.

Most recently, Bae et al. (2016) equipped a slaughterhouse with an AutoFOM system in Dodram LPC (Gyeonggi province of Korea) to predict the lean meat yield from the ham cut of pig carcasses. In the present study, we used a VCS2000 (E+V Technology GmbH, Oranienburg, Germany) for the rapid and on-line estimation of the LMP of pork carcasses. The VCS2000 is a picture-processing (video image analysis) system that is used to automatically determine the trade value of pork half carcasses, as well as to calculate the lean meat of the primal cuts of the carcass (i.e., ham, tenderloin, loin, neck, shoulder, belly, ribs etc.) (Daumas and Causeur, 2008; Engel et al., 2006; Font I Furnols and Gispert, 2009). By using the VCS2000 system, we believe that the quality of domestic pigs can be improved by on-line measurements of the back-fat thickness and the LMP of the major cuts, where the results will enable pork producers to produce pork of higher market value.

\section{Conclusions}

The present study utilizes a VCS2000 image processing-based technique for the prediction of LMP in Korean pork 
carcasses. The results of the present study illustrate the ability of the developed equation to accurately predict LMP in the whole carcass. In addition, prediction of lean meat in major cuts is also possible with the technique used in the present study, with a high degree of accuracy and a low prediction error. The results of this study will provide the basis for a fairer payment system for producers of pork, while the recently increasing demand for pork consumption can be met more efficiently in Korea.

\section{Acknowledgements}

This work was partially supported by Korea Institute of Planning and Evaluation for Technology in Food, Agriculture, Forestry and Fisheries (IPET) through Agriculture, Food and Rural Affairs Research Center Support Program, funded by Ministry of Agriculture, Food and Rural Affairs (MAFRA), Korea (No. 717001071WT111).

\section{References}

Bae JG, Lee YK, Park BY, Lym HS, Jung BS. 2016. Prediction of ham weight with the autofom in Korea. Korean J Vet Serv 39:7-12.

Branscheid W, Dobrowolsk A. 2000. Evaluation of market value: Comparison between different techniques applied on pork carcasses (short communication). Arch Anim Breed 43:131-137.

Choe JH, Yang HS, Lee SH, Go GW. 2015. Characteristics of pork belly consumption in South Korea and their health implication. J Anim Sci Technol 57:1-7.

Daumas G, Causeur D. 2008. Tests d'homologation des appareils automatiques de classement des carcasses de porc. Journées Rech Porc 40:91-92.

Engel B, Lambooij E, Buist WG, Reimert H, Mateman G. 2006. Prediction of the percentage lean of pig carcasses with a small or a large number of instrumental carcass measurements - An illustration with HGP and vision. Anim Sci 82:919928.

European Commission. 2014. Commission Regulation (EC) 1249/2008 of 10 December 2008: Laying down detailed rules on the implementation of the community scales for the classification of beef, pig and sheep carcases and the reporting of prices thereof. Available from: https://publications.europa.eu/en/publication-detail///publication/9716803a-8887-49569877-629031 ec7723/language-en. Accessed at: Oct 13, 2018.

Font I Furnols M, Engel B, Gispert M. 2004. Validation of the Spanish equation to predict the lean meat percentage of pig carcasses with the Fat-O-Meat'er. Spanish J Agric Res 2:545-549.

Font I Furnols M, Gispert M. 2009. Comparison of different devices for predicting the lean meat percentage of pig carcasses. Meat Sci 83:443-446.

Forrest JC, Kuei CH, Orcutt MW, Schinckel AP, Stouffer JR, Judge MD. 1989. A review of potential new methods of on-line pork carcass evaluation. J Anim Sci 67:2164-2170.

Gispert M, Font I Furnols M. 2012. The use of pig carcass grading devices and computer tomography for predicting lean meat in the carcass and the main joints. $58^{\text {th }}$ International Congress of Meat Science and Technology, Canada. pp 1-3.

Kim GW, Kim HY. 2018. Physicochemical properties of M. longissimus dorsi of Korean native pigs. J Anim Sci Technol 60:6.

Lucas D, Brun A, Gispert M, Carabús A, Soler J, Tibau J, Font-i-Furnols M. 2017. Relatioship between pig carcass 
characteristics measured in live pigs or carcasses with piglog, fat-o-meat'er and computed tomography. Livest Sci 197: 88-95.

Nissen PM, Busk H, Oksama M, Seynaeve M, Gispert M, Walstra P, Hansson I, Olsen E. 2006. The estimated accuracy of the EU reference dissection method for pig carcass classification. Meat Sci 73:22-28.

Olsen EV, Potokar MC, Oksama M, Kien S, Lisiak D, Busk H. 2007. On-line measurements in pig carcass classification: Repeatbility and variation caused by operator and the copy of instrument. Meat Sci 75:29-38.

Rahman SMAK, Sathik MM, Kannan KS. 2012. Multiple linear regression model in outlier detection. Int J Res Comput Sci 2:23-28.

Savescu RF, Laba M. 2016. Multivariate regression analysis applied to the calibration of equipment used in pig meat classification in Romania. Meat Sci 116:16-25. 